\title{
Neuromelanin, neurotransmitter status and brainstem location determine the differential vulnerability of catecholaminergic neurons to mitochondrial DNA deletions
}

Matthias Elstner ${ }^{1,2}$, Sarina K Müller ${ }^{1}$, Lars Leidolt ${ }^{1}$, Christoph Laub ${ }^{1,2}$, Lena Krieg ${ }^{1}$, Falk Schlaudraff ${ }^{3}$, Birgit Liss ${ }^{3}$, Chris Morris ${ }^{4,5}$, Douglass M Turnbull ${ }^{4,6}$, Eliezer Masliah ${ }^{7}$, Holger Prokisch ${ }^{8,9}$, Thomas Klopstock ${ }^{1,2+}$ and Andreas Bender ${ }^{1,10^{*}+}$

\begin{abstract}
Background: Deletions of the mitochondrial DNA (mtDNA) accumulate to high levels in dopaminergic neurons of the substantia nigra pars compacta (SNc) in normal aging and in patients with Parkinson's disease (PD). Human nigral neurons characteristically contain the pigment neuromelanin (NM), which is believed to alter the cellular redox-status. The impact of neuronal pigmentation, neurotransmitter status and brainstem location on the susceptibility to mtDNA damage remains unclear. We quantified mtDNA deletions ( $\triangle m t D N A)$ in single pigmented and non-pigmented catecholaminergic, as well as non-catecholaminergic neurons of the human SNc, the ventral tegmental area (VTA) and the locus coeruleus (LC), using laser capture microdissection and single-cell real-time PCR.

Results: In healthy aged individuals, $\triangle \mathrm{mtDNA}$ levels were highest in pigmented catecholaminergic neurons (25.2 \pm $14.9 \%)$, followed by non-pigmented catecholamergic (18.0 $\pm 11.2 \%)$ and non-catecholaminergic neurons (12.3 \pm 12.3\%; $\mathrm{p}<0.001)$. Within the catecholaminergic population, $\triangle \mathrm{mtDNA}$ levels were highest in dopaminergic neurons of the SNc (33.9 $\pm 21.6 \%)$ followed by dopaminergic neurons of the VTA $(21.9 \pm 12.3 \%)$ and noradrenergic neurons of the LC $(11.1 \pm 11.4 \% ; \mathrm{p}<0.001)$. In PD patients, there was a trend to an elevated mutation load in surviving non-pigmented nigral neurons $(27.13 \pm 16.73)$ compared to age-matched controls (19.15 $\pm 11.06 ; p=0.052)$, but levels where similar in pigmented nigral neurons of PD patients (41.62 \pm 19.61$)$ and controls (41.80 \pm 22.62$)$.

Conclusions: Catecholaminergic brainstem neurons are differentially susceptible to mtDNA damage. Pigmented dopaminergic neurons of the SNc show the highest $\triangle \mathrm{mtDNA}$ levels, possibly explaining the exceptional vulnerability of the nigro-striatal system in PD and aging. Although loss of pigmented noradrenergic LC neurons also is an early feature of PD pathology, mtDNA levels are not elevated in this nucleus in healthy controls. Thus, $\triangle m t D N A$ are neither an inevitable consequence of catecholamine metabolism nor a universal explanation for the regional vulnerability seen in $\mathrm{PD}$.
\end{abstract}

Keywords: Parkinson disease, aging, neurodegeneration, catecholaminergic neurons, mitochondrial DNA, single neuron analysis, laser-microdissection

\footnotetext{
* Correspondence: andreas.bender@med.uni-muenchen.de

† Contributed equally

'Department of Neurology with Friedrich-Baur-Institute, Ludwig-Maximilians-

University, 81377 Munich, Germany

Full list of author information is available at the end of the article
} 


\section{Background}

Oxidative stress and mitochondrial dysfunction are believed to have a dominant role in mechanisms of aging and neurodegenerative disorders such as Parkinson disease (PD) [1]. The mitochondrial theory of aging proposes that production of reactive oxygen species (ROS) in mitochondria causes accumulating damage to proteins, lipids, and mitochondrial DNA (mtDNA). As a consequence, mitochondrial dysfunction and ROS production may build up in a vicious cycle that eventually results in cell death $[2,3]$. Damage to mtDNA is central to this theory and early studies provide evidence for the accumulation of somatic mtDNA deletions $(\triangle \mathrm{mtDNA})$ in aging postmitotic tissues with high energy demand, such as skeletal muscle and the brain $[4,5]$. Nevertheless, due to the low abundance of $\triangle$ mtDNA detected in crude tissue homogenates, their functional significance remained controversial. By combining laser-microdissection (LMD) with a quantitative realtime PCR (RT-PCR) assay, we demonstrated the agerelated accumulation of clonally expanded $\Delta$ mtDNA in individual post mortem dopaminergic neurons of the substantia nigra pars compacta (SNc) [6]. Indicative of a resulting functionally relevant biochemical defect, neurons with high levels $(\sim 60 \%) \triangle$ mtDNA had mitochondrial cytochrome-c oxidase (COX, complex IV of the mitochondrial respiratoy chain) deficiency on histochemical examination in PD patients and controls. These findings were independently confirmed using a different methodological approach [7]. Our studies into the regional distribution of $\Delta$ mtDNA further showed that dopaminergic nigral neurons have a higher propensity to accumulate $\triangle \mathrm{mtDNA}$ than extranigral populations, e.g. in the putamen, the hippocampus or the frontal cortex $[8,9]$. Besides their catecholaminergic neurotransmitter status, a prominent feature of these neurons is their pigmentation, i.e. the intraneuronal accumulation of neuromelanin (NM). NM has long been considered a cellular waste product via the non-enzymatic oxidization of dopamine or other catecholamines, but some evidence points towards a regulated production that might involve alpha-synuclein $[10,11]$. Its contribution to neurodegenerative processes is far from understood as there is evidence for both neuroprotective and neurotoxic properties [12-14]. As a possible mechanism, it has been proposed that NM might serve to control iron homeostasis within pigmented neurons [15]. If the iron chelation ability of NM is reduced, increased levels of intra-neuronal free iron may stimulate ROS production. In $\mathrm{PD}$, pigmented neurons contain less NM than in healthy brains, while the optical density of the pigment is increased $[16,17]$. These changes of neuronal NM content and composition may cause a loss of protective properties [18]. Indeed, we have previously shown that individual dopaminergic neurons have elevated iron levels in PD
[19]. Thus, increased ROS generation, mtDNA mutations and mitochondrial dysfunction might pave the way for neurodegenerative processes in PD [20].

In this context the question arises, if location, neurotransmitter status or the presence of NM determines the vulnerability of dopaminergic nigral neurons to accumulate mtDNA damage. Herein, we investigated the association of these factors with mtDNA levels in single post mortem catecholaminergic neurons that were dissected from the SNc, the ventral tegmental area (VTA) and the locus coeruleus (LC) of post mortem brains of healthy aged individuals and PD patients.

\section{Results}

\section{Association of pigmentation and $\triangle$ mtDNA levels}

In the adult human brain, NM is easily identifiable as a black-brown pigment by light microscopy. NM-containing neurons are distributed throughout the entire brainstem, but the largest clusters are found in the SNc, the VTA and the LC [18]. In a pilot experiment, we randomly collected pigmented neurons from the SNc of five healthy controls $(80.8 \pm 8.6 y)$ and compared $\triangle \mathrm{mtDNA}$ levels to those seen in non-pigmented neurons within the same specimens. We found that non-pigmented neurons had a mean of $31.0 \pm 25.1 \% \triangle \mathrm{mtDNA}$, whereas pigmented neurons had a mean of $49.2 \% \pm 18.3 \% \Delta \mathrm{mtDNA}(\mathrm{p}=0.017)$. This preliminary data suggested that an increased vulnerability is associated with NM-pigmentation of midbrain neurons in healthy aged controls.

Encouraged by these results, we next established an immunohistochemical staining protocol to facilitate a more specific and extensive analysis of catecholaminergic neurons of the SNc, the VTA and the LC. Using a successive $\mathrm{TH} / \mathrm{NeuN}$ antibody labeling and DAB/HRP reaction, catecholaminergic $\left(\mathrm{TH}^{+}\right)$neurons were positively discriminated from non-catecholaminergic $\left(\mathrm{TH}^{-}\right)$neurons (e.g. GABAergic interneurons) and from glia cells. $\mathrm{TH}^{+} \mathrm{immu}-$ noreactivity results in a brown cytosolic reaction product, while TH- neurons remain unstained. NeuN immunoreactivity results in a grey appearance of $\mathrm{TH}^{-}$neurons due to the successive DAB/Nickel reaction. Pigmented and nonpigmented neurons were further distinguished by the visible presence or absence of NM (Figure 1). For these studies, we extended the analysis with an independent group of 19 controls $(68.8 \pm 19.4 \mathrm{y})$ and 14 PD specimens $(75.1 \pm 7.8 \mathrm{y})$.

We first asked, whether our initial data could be validated after immunhistochemical identification of catecholaminergic neurons. To this end, we analyzed $\triangle$ mtDNA levels of $\mathrm{TH}^{+}$neurons captured from all three regions (SN, VTA and LC). In a combined analysis of all regions, we confirmed a significant difference of mtDNA levels between non-pigmented $(18.0 \% \pm 11.2 \%)$ 


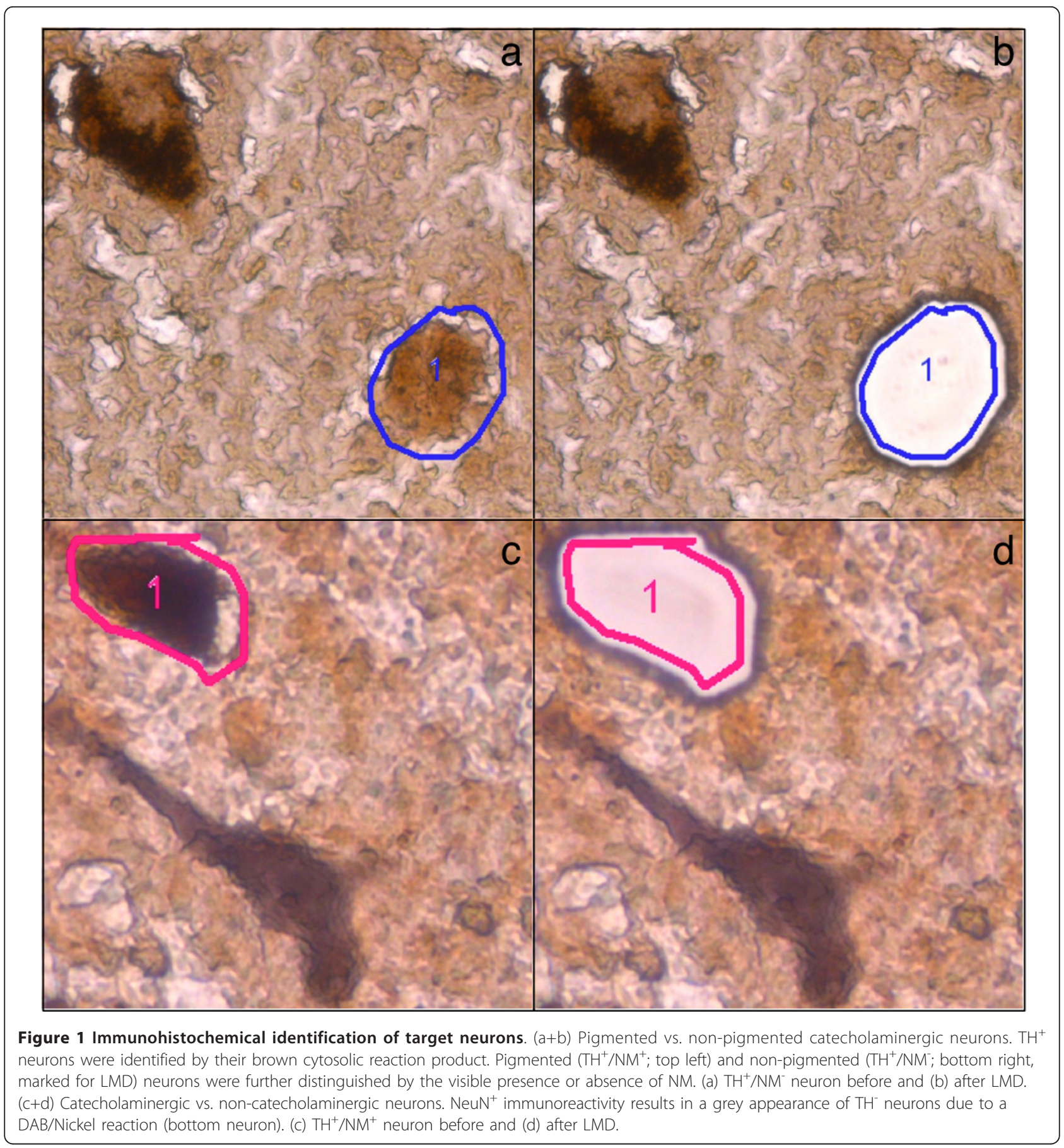

and pigmented neurons $(25.2 \% \pm 14.9 \%$; $\mathrm{p}=0.003)$. Thus, in catecholaminergic brainstem neurons, the presence of NM is associated with higher $\triangle \mathrm{mtDNA}$ levels, independent of location and dopaminergic or noradrenergic neurotransmitter status.

\section{Influence of neurotransmitter status on $\triangle$ mtDNA levels}

We next asked whether $\triangle \mathrm{mtDNA}$ levels are generally higher in $\mathrm{TH}^{+}$(catecholaminergic) compared to $\mathrm{TH}^{-}$ neurons, i.e. neurons that contain neither dopamine nor noradrenaline. For this analysis, $\mathrm{TH}^{-} / \mathrm{NeuN}^{+}$neurons were additionally sampled from SN, VTA and LC. RTPCR data showed that TH-negative midbrain neurons had low mtDNA levels of $12.3 \% \pm 12.3$. After combining this and the previous data for statistical analysis, we found a significant difference between $\mathrm{TH}^{-}$neurons and pigmented as well as non-pigmented $\mathrm{TH}^{+}$neurons (Mann-Whitney-Test; $\mathrm{p}=0.005$ ). In these three groups, 
levels of mtDNA increase in the order (Kruskal-WallisTest; $\mathrm{p}<0.001)$ : non-catecholaminergic $(12.3 \%)<$ catecholaminergic/non-pigmented $(18.0 \%)<$ catecholaminergic/pigmented (25.2\%) (Figure 2).

\section{Vulnerability of SNc, VTA and LC neurons}

The SNc and VTA both predominately contain dopaminergic neurons, while the LC contains noradrenergic neurons. In the $\mathrm{SNc}$ and in the $\mathrm{LC}$ the pigment is found in approximately $95 \%$ of neurons, whereas in the VTA only about $50 \%$ neurons are pigmented [11]. We therefore asked, to what extend the location has an impact on deletion levels independent of neuronal pigmentation. Combined analysis of pigmented and non-pigmented $\mathrm{TH}^{+}$neurons showed an average of $11.1 \pm 11.4 \% \Delta \mathrm{mtDNA}$ in the LC, $21.9 \pm 12.3 \%$ in the VTA and $33.9 \pm 21.6 \%$ in the SNc. Interregional differences were significant between all groups at $\mathrm{p}<0.001$ (Figure 3). Thus, dopaminergic neurons of the SNc are more susceptible to $\triangle \mathrm{mtDNA}$ than those of the VTA and both are more susceptible than noradrenergic neurons of the $\mathrm{LC}$.

\section{$\Delta$ mtDNA levels in PD nigral neurons}

Lastly, we quantified $\Delta$ mtDNA levels in PD cases $(n=14$; mean age $75.1 \pm 7.8$ years). Collection and analysis was restricted to $\mathrm{SN}$ dopaminergic neurons due to the paucity of suitable tissue samples. In PD, $\Delta$ mtDNA levels of

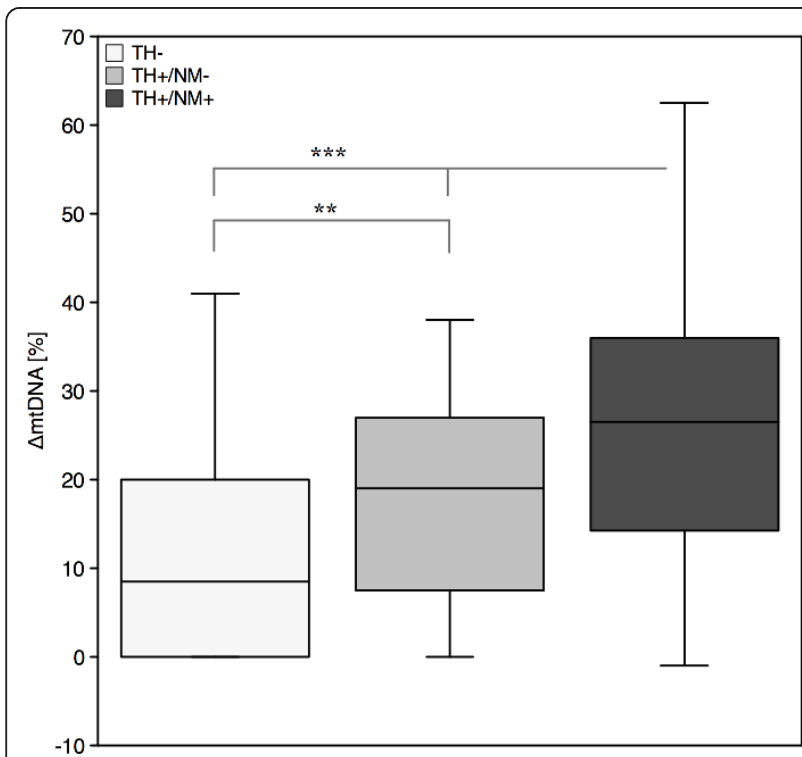

Figure 2 Differential susceptibility of catecholaminergic pigmented neurons to deletions of mitochondrial DNA. Levels of $\triangle \mathrm{mtDNA}$ raise in the order of non-catecholaminergic $\left(\mathrm{TH}^{-} / \mathrm{NeuN}^{+}\right)$ $<$ catecholaminergic/non-pigmented $\left(\mathrm{TH}^{+} / \mathrm{NM}^{-}\right)<$

catecholaminergic/pigmented neurons $\left(\mathrm{TH}^{+} / \mathrm{NM}^{+}\right)$. Differences were significant at $p=0.005$ (**; Mann-Whitney-Test) and $p=0.001$ (***; Kruskal-Wallis-Test).

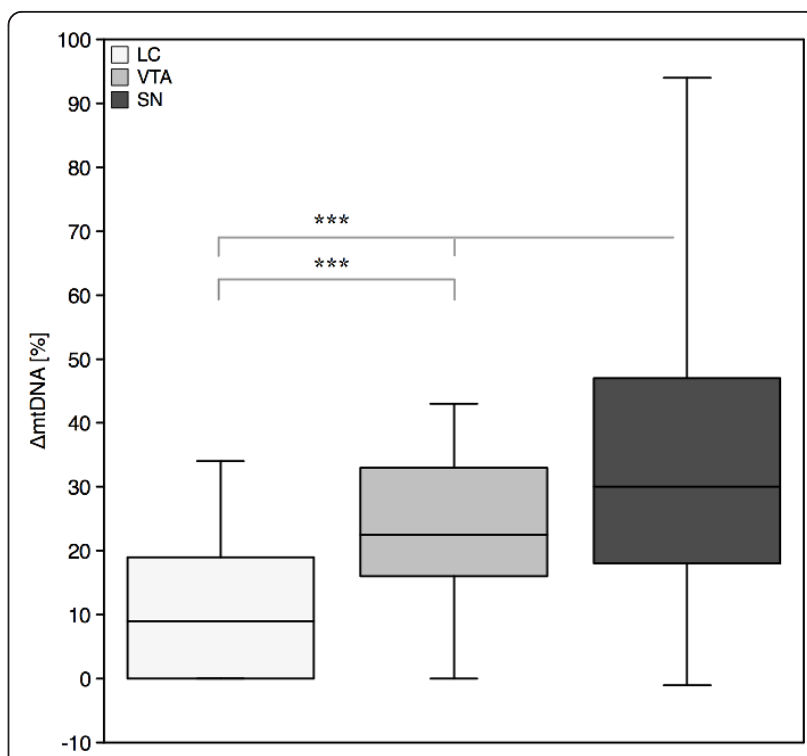

Figure 3 Differential vulnerability of SNc, VTA and LC neurons to deletions of mitochondrial DNA. Lowest levels were seen for noradrenergic neurons of the LC, followed by dopaminergic neurons of the VTA and the SNc. Differences were significant at $p=$ 0.001 (***; Kruskal-Wallis-Test).

pigmented neurons $(41.62 \pm 19.61)$ were again higher than those of non-pigmented neurons $(27.13 \pm 16.73$; $\mathrm{p}=$ 9.6E-05), thus independently reproducing the results seen in the control group. We then compared $\Delta$ mtDNA levels in PD cases to those seen in age-matched controls ( $\mathrm{n}=19$; mean age $78.7 \pm 9.0$ years). In non-pigmented neurons there was a trend to higher deletions in PD vs. controls $(\mathrm{PD}=27.13 \pm 16.73$; controls $=19.15 \pm 11.06$; $\mathrm{p}=0.052)$. No difference was seen for pigmented neurons in PD (41.62 \pm 19.61$)$ and controls (41.80 \pm 22.62 ; Figure 4).

\section{Differential vulnerability of catecholaminergic brainstem nuclei in healthy aging}

To generate a concise picture of $\Delta \mathrm{mtDNA}$ levels in the aged human brain, we extracted data coming from all control individuals over 60 years of age $(n=19$; mean age $78.7 \pm 9.0$ years). In our synopsis, individual levels of $\triangle \mathrm{mtDNA}$ in relation to brainstem location, pigmentation and neurotransmitter status are illustrated (Figure 5). This data underlines the prominent vulnerability of pigmented nigral neurons, followed by pigmented neurons of the VTA and non-pigmented neurons of both nuclei, whereas the LC has overall low deletion levels.

\section{Discussion}

Degeneration of pigmented dopaminergic neurons is one of the neuropathological hallmarks of PD [21,22]. In these neurons, ROS generation may be enhanced by the 


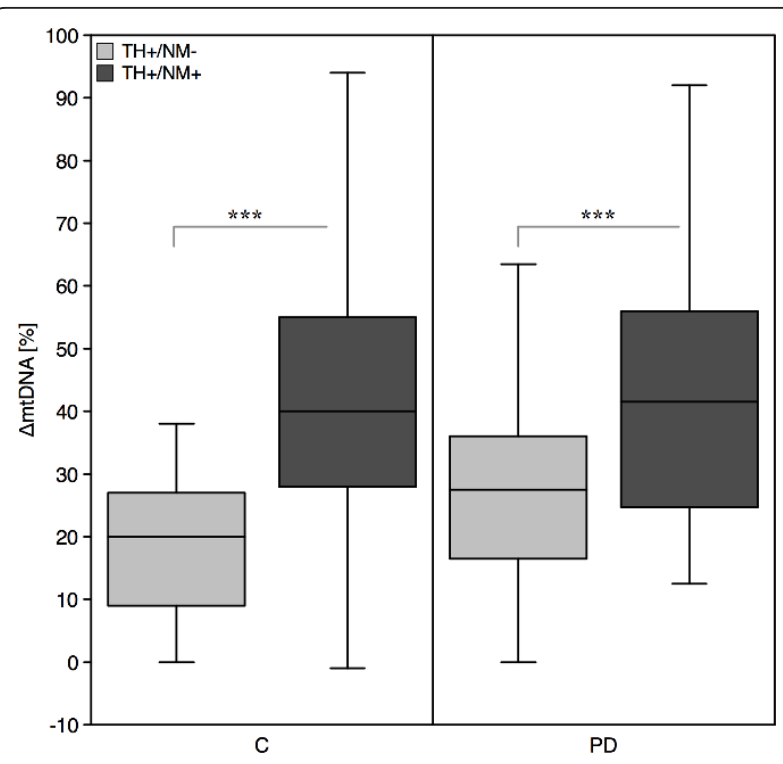

Figure 4 Levels of mitochondrial DNA deletions in nigral neurons of PD and controls. Pigmented neurons $\left(\mathrm{TH}^{+} / \mathrm{NM}^{+}\right)$of the SNc have considerably higher $\triangle \mathrm{mtDNA}$ levels than non-pigmented neurons $\left(\mathrm{TH}^{+} / \mathrm{NM}^{-}\right)$in controls $(\mathrm{C})$ and $\mathrm{PD}\left({ }^{* *} \mathrm{p}=0.001\right)$. In nonpigmented neurons there was a trend to higher deletions in PD vs. controls $(p=0.052)$.

presence of autooxidizable dopamine, low glutathion and high iron content [23]. Additionally, recent data has revealed the reliance on $\mathrm{Ca}^{2+}$ channels to maintain autonomous activity in aging dopaminergic neurons, causing sustained metabolic stress on mitochondria [24]. Decreased expression of nuclear encoded mitochondrial genes and of genes in energy-sensing pathways might further aggravate mitochondrial dysfunction [25]. The combination of these and other factors likely accelerates cellular aging processes and propagates clonal expansion of mtDNA, which are believed to arise during the repair of oxidatively damaged mtDNA $[6,26,27]$. COX-deficient neurons are also found in hippocampal neurons of aged individuals and Alzheimer's patients, but dopaminergic neurons of the SNc accumulate deletions to considerably higher levels $[8,9]$. These neurons are characterized by the age-related appearance and accumulation of NMpigment. In vitro data provides an intriguing view on the potential protective and harmful properties of NM, which might change depending on the melanin species, its protein component, sulfhydryl residues and the cellular redox-state. Depletion of glutathione and upregulation of glutathione peroxidase activity in response to oxidative stress may further drive the production of neuromelanin [28]. While NM can cause apoptosis of DA neurons through an impact on mitochondrial redox state and S-glutathionylation [29], a protective role was shown in primary mesencephalic neurons under conditions of high oxidative load [30].

\section{Increased $\triangle \mathrm{mtDNA}$ levels in pigmented neurons}

To further elucidate the relationship of pigmentation and ROS damage in the human brain, we determined $\triangle \mathrm{mtDNA}$ levels by RT-PCR analysis of single human post mortem brainstem neurons that were obtained from control individuals and PD patients by immunohistochemical characterization and LMD. The primary finding of this study is that $\triangle \mathrm{mtDNA}$ levels are reproducibly elevated in neurons containing NM compared to non-pigmented neurons. To our knowledge, these data establish for the first time an association of neuronal pigmentation and mtDNA damage in human post mortem brain. Due to the limitations of a post mortem study, a causal relationship is difficult to prove or exclude. On the one hand, NM itself might cause increased ROS and mtDNA damage leading to the clonal expansion of $\triangle \mathrm{mtDNA}$. On the other hand, an independent pathophysiological mechanism may impose cellular stress on these neurons and cause increase in both, $\triangle \mathrm{mtDNA}$ levels and NM content.

\section{Only dopaminergic neurons show elevated $\triangle \mathrm{mtDNA}$ levels}

In the literature, several lines of evidence argue against the notion that NM is a simple degradation product of catecholamine transmitter metabolism. On the contrary, NM synthesis and turnover may underlie a yet to determined enzymatic regulatory process, possibly involving alphasynuclein $[11,17,31]$. While NM is found in most dopaminergic neurons of the $\mathrm{SN}$ and noradrenergic neurons of the LC, it does not develop in dopaminergic neurons of the olfactory bulb, some hypothalamic nuclei, nor in medullary adrenergic neurons [32]. Moreover, despite the fact that $\mathrm{TH}$ is the rate limiting enzyme in catecholamine synthesis there is no clear correlation between the degree of NM pigmentation and TH immunoreactivity [33,34]. We therefore asked, whether $\Delta \mathrm{mtDNA}$ levels are generally higher in catecholaminergic than in non-catecholaminergic neurons. We found that in noradrenergic neurons of the LC, $\Delta$ mtDNA were not elevated compared to THneurons. In contrast to this, dopaminergic neurons of the SNc and VTA have considerably elevated $\triangle \mathrm{mtDNA}$ levels (Figure 2) compared to other neuronal populations.

\section{SNc neurons are highly susceptible to $\triangle$ mtDNA - LC neurons are not}

During PD disease-progression, intraneuronal pathology and neurodegeneration are seen in an 'ascending' pattern throughout the entire brain (i.e. Braak stages) [21]. Within the brainstem, catecholaminergic nuclei display a differential pathology: whereas the LC is affected early in disease and degeneration is reported to exceed that of the SNc [35], neuronal survival in the VTA is considerably higher even in severe cases [36,37]. We therefore sought to determine mtDNA mutation load in these 




Figure 5 Differential vulnerability of catecholaminergic brainstem neurons in healthy aged controls. 3-D model of the brain showing location of brainstem nuclei and $\triangle \mathrm{mtDNA}$ levels of pigmented $\left(\mathrm{TH}^{+} / \mathrm{NM}^{+}\right)$and non-pigmented $\left(\mathrm{TH}^{+} / \mathrm{NM}^{-}\right)$catecholaminergic neurons, as well as non-catecholaminergic $\left(\mathrm{TH}^{-}\right)$neurons in these nuclei. Values represent mean \pm standard deviation of data collected from healthy aged controls (78.7 \pm 9.0 years). Highest deletion levels are seen in pigmented neurons of the SNc (black). VTA neurons show intermediate $\triangle m$ mtDNA levels (grey) and LC neurons lowest (light grey). PD pathology is deviating from this pattern, as SNc and LC show heavy degeneration whereas the VTA is relatively spared.

nuclei in healthy controls. If $\triangle \mathrm{mtDNA}$ played an integral part in the selective vulnerability in $\mathrm{PD}$, a regional pattern of $\Delta$ mtDNA resembling that of Braak stages might be seen. Contrary to these expections, we found that deletion levels were considerably higher in SNc neurons than in the similarly pigmented LC neurons, (Figure 3). This raises doubts about a causative continuity of catecholamine metabolism > NMpigmentation > ROS generation $>$ mtDNA damage. Thus, $\triangle$ mtDNA levels are not tightly associated with the differential neuronal vulnerability seen in PD (Figure 5). This finding argues against the notion that PD pathology is an acceleration of molecular events found in healthy aging, which has also been shown in morphometric studies [34]. While the agedependent loss of DA neurons is a linear process and mainly affects the dorsal part of the SNc (6,9\% loss per 
decade), followed by the medial ventral part (5,4\% per decade) and nearly sparing the ventral part (2,1\% loss per decade), neurodegeneration in PD is rapidly progressive with an overall loss of DA neurons of $45 \%$ in the first decade. Furthermore, the ventral part of the SNc is the most affected part in this progress with an average loss of $95 \%$, followed by the medial ventral part $(71 \%)$ and the dorsal part (56\%). It has to be stressed that - due to tissue limitations - we were not able to analyze the regional distribution of $\triangle \mathrm{mtDNA}$ in PD samples to the extent done for controls. Therefore, we cannot rule out that $\triangle \mathrm{mtDNA}$ levels increase to significant levels in the LC or other neuronal populations affected in PD.

\section{Elevated $\triangle \mathrm{mtDNA}$ levels in SNc of PD}

We further asked, whether $\triangle \mathrm{mtDNA}$ levels reflect disease pathology in PD as was suggested by previous studies [6]. Results from PD cases independently confirmed higher $\triangle \mathrm{mtDNA}$ levels in pigmented vs. non-pigmented neurons. Compared to controls, we found a not significant trend to higher $\triangle \mathrm{mtDNA}$ levels in non-pigmented SNc neurons of PD brains, but this was not seen for pigmented neurons (Figure 4). The interpretation of this data is complicated by the extensive and differential neuronal loss seen in PD brains. Most severely affected neurons might have already been lost and probably only well protected and 'more resistant' neurons were left for sampling. Furthermore, within the SNc, a regional vulnerability is seen for PD: nigral neurons of the lightly pigmented ventral tier degenerate first, while the heavily pigmented of the dorsal tier are relatively preserved $[16,34,38]$. If pigmented neurons are mainly sampled from the pool of 'resistant' neurons located in the dorsal tier this might result in similar $\triangle \mathrm{mtDNA}$ levels comparing PD and controls. In this study, the available midbrain samples did not allow for the discrimination of regional differences in $\mathrm{PD}$, but this is planned for future studies.

Following a different line of argumentation, $\triangle \mathrm{mtDNA}$ may simply play no relevant role in the pathogenesis of PD. Contradicting this notion, high $\triangle \mathrm{mtDNA}$ levels are associated with a clear biochemical defect in nigral neurons and the percentage of these COX-deficient neurons is increased in the SN of PD patients [6]. Furthermore, gene defects of the mtDNA polymerase $\gamma$ (POLG) result in the accumulation of $\triangle \mathrm{mtDNA}$ and can cause parkinsonism [39]. Importantly, breakpoint analysis revealed that the types of $\triangle \mathrm{mtDNA}$ that have clonally expanded in nigra neurons from PD patients and age-matched controls are similar to those from a patient with POLG mutations who had parkinsonism [40].

This study shows that $\triangle \mathrm{mtDNA}$ are a common agerelated phenomenon in pigmented nigral neurons, both in PD patients and healthy individuals. Thus, accumulation of $\triangle \mathrm{mtDNA}$ clearly cannot serve as the single explanation for neurodegeneration in $\mathrm{PD}$, but may precipitate dopamine neuron death in combination with other endogenous and exogenous factors. Interestingly, recent data implies that age-related accumulation of NM-pigment might induce $\alpha$-synuclein expression, another important factor determining PD pathology [41].

\section{Conclusions}

NM formation and turnover might constitute a protective system regulating neuronal redox state $[11,18]$. On the other hand, NM may contribute to ROS generation through release of redox-active iron under certain pathological conditions [15]. In this study, we found increased $\triangle \mathrm{mtDNA}$ levels in pigmented midbrain neurons, which is consistent with both theories. Dopaminergic neurons have elevated levels of $\triangle \mathrm{mtDNA}$, supporting the role of dopamine metabolism in the generation of ROS and mtDNA damage. Importantly, in noradrenergic neurons, a causative relation of pigmentation, production of ROS and accumulation of $\triangle \mathrm{mtDNA}$ cannot be established. Since the LC is affected early and severely in PD pathology, different factors must account for the vulnerability of catecholamingergic neurons in this nucleus.

\section{Methods}

\section{Ethics statement}

Frozen midbrain tissue was requested from the Newcastle Brain Tissue Resource and the German brain bank $\left(\right.$ Brain-Net ${ }^{\mathbb{B}}$ ). Written consent was obtained with verification/assent in writing from next of kin who confirmed the wishes at time of death. All procedures were in line with the UK Human Tissue Authority guidance and approved by the Local Research Ethics Committee. The study is in accordance with the ethical standards laid down in the 1964 Declaration of Helsinki.

\section{Patients and controls}

Control individuals had no prior history of neurological disease $(\mathrm{n}=24$, mean age $75.1 \pm 7.8$ years, post mortem interval (PMI) $24.0 \pm 9.0$ hours). Patients had a clinical and neuropathological diagnosis of PD $(\mathrm{n}=14$, mean age $71.3 \pm 18.2$ years, PMI $32.1 \pm 18.7$ hours). Neither age $(\mathrm{p}=0.24)$ nor PMI $(\mathrm{p}=0.1)$ were significantly different between groups. Neuropathological examination had demonstrated the presence of Lewy body pathology in the substantia nigra with typical pathological features, including moderate to severe neuronal loss and gliosis. Synuclein immunohistochemistry or ubiquitin immunohistochemistry was used to confirm findings from $\mathrm{H} \& \mathrm{E}$ stained sections and cases were graded according to published criteria for Lewy body disorders (LBD) $[21,42]$. 


\section{Histology and immunohistochemistry}

Unfixed human midbrains stored at $-80^{\circ} \mathrm{C}$ were used for analysis. $20 \mu \mathrm{m}$ sections were cut and mounted onto Leica $2 \mu \mathrm{m}$ PEN membrane slides (Leica Microsystems, Wetzlar, Germany) prior to staining and microdissection. For pilot experiments ( $\mathrm{n}=5$ controls), sections were stained with cresyl violet (Merck, Darmstadt, Germany) and dehydrated in an ethanol series. For most experiments, a successive Tyrosin-Hydroxylase (TH) and Neuronal Nuclei (NeuN) double-staining was applied. In detail, frozen sections were fixed in ice-chilled acetone for 7 minutes and air-dried for 10 minutes at room temperature (RT). All solutions were prepared in phosphate buffered saline 1\% Triton X-100 (PBST) and all incubations were performed at RT. Sections were washed twice in PBST and blocked with 5\% normal goat serum (NGS) for $30 \mathrm{~min}$. An anti-mouse poly horseradish peroxidase (HRP) kit was used for detection of primary antibodies according to the manufacturers protocol (Millipore, Billerica, MA, USA). Primary rabbit anti-TH antibody (abcam, Cambridge, UK) was applied at 1:300 in PBST with 5\% NGS for $60 \mathrm{~min}$ and staining was developed with the DAB Chromogen-Buffer provided with the kit for $5 \mathrm{~min}$. Sections were washed in tap water followed by blocking and incubation with 1:400 mouse IgG1 antiNeuN (Millipore, Billerica, MA, USA) for 45 min. For successive NeuN detection, nickel chloride was added to the DAB substrate solution and developed for $10 \mathrm{~min}$. The addition of nickel chloride produces a dark grey appearance of $\mathrm{NeuN}^{+}$neurons, thereby enabling distinction from the light brown of DAB-mediated $\mathrm{TH}^{+}$immunolabelling. Finally, sections were washed, dehydrated in $100 \%$ ethanol and air-dried for $30 \mathrm{~min}$. Membrane sections were used for LMD immediately or frozen at $-20^{\circ} \mathrm{C}$ for later use.

\section{Quantification of $\triangle \mathrm{mtDNA}$}

UV-Laser-microdissection was performed on a Leica LMD6000 microscope (Leica, Wetzlar, Germany). Single neurons were collected into separate reaction tubes and DNA was extracted with the DNA Micro Kit (Qiagen, Düsseldorf, Germany), according to the manufacturer's protocol. Quantification of $\triangle \mathrm{mtDNA}$ levels was based on the RT-PCR method previously described, using relative quantification of the mitochondrial ND1 and ND4 genes by means of the delta-delta-CT-method [6]. Differing from the original method, we further optimized the RTPCR assay to be run as a duplex experiment with quantification of ND1 and ND4 genes within the same reaction. Using this protocol, there is high correlation with deletion quantification by southern blot and by the original method [43]. The following primers (MWG Biotech, Ebersberg, Germany) and TaqMan probes (Life
Technologies, Carlsbad, CA, USA) were used: ND1 (forward primer nt 3485-3504, reverse primer nt 3553-3532; VIC-labeled probe nt 3506-3529) and ND4 (forward primer nt 12087-12109, reverse primer nt 12170-12140, FAM-labeled probe nt 12111-12138). Final concentrations were $900 \mathrm{nM}$ for primers and $250 \mathrm{nM}$ for probes. The Taqman Universal PCR Mastermix (Life Technologies, Carlsbad, CA, USA) was used for the assay in a 25 $\mu \mathrm{l}$ reaction mix per sample. Experiments were performed on an Applied Biosystems StepOnePlusTM system (Life Technologies, Carlsbad, CA, USA). Standard cycling conditions were used as follows: activation for 2 minutes @ $50^{\circ} \mathrm{C}$ followed by 10 minutes @ $95^{\circ} \mathrm{C}$; PCR (40 cycles) for 15 seconds @ $95^{\circ} \mathrm{C}$ followed by 1 minute at $60^{\circ} \mathrm{C}$. Samples were analyzed in triplicates and the resulting mean values were used for statistical analysis.

\section{Statistical analysis and graphic design}

All statistical analyses were performed with SPSS 18.0 for Mac (PASW Statistics, IBM). A total of 383 neurons were captured and mtDNA deletion levels were analyzed for every single neuron. Data of all neurons was available for analysis. Since the mtDNA deletion values did not have a normal distribution (Kolmogorov-Smirnov-Test $\mathrm{p}=$ 0.007), we performed non-parametric tests for all statistical procedures (Mann-Whitney-Test and Kruskal-Wallis one-way analysis of variance). Values are expressed as mean \pm standard deviation. Box plots show median, lower and upper quartile. Whiskers are extended to extreme data points. Figure 5 was generated using Cinema 4D (Maxon, Friedrichsdorf, Germany).

\section{List of abbreviations}

mtDNA: mitochondrial DNA; SNc: substantia nigra pars compacta; VTA: ventral tegmental area; LC: locus coeruleus; PD: Parkinson's disease; NM: neuromelanin; mtDNA: mtDNA deletions; ROS: reactive oxygen species; LMD: laser-microdissection; $\mathrm{TH}$ : tyrosine hydroxylase; COX: cytochrome-c oxidase; PMl: post mortem interval.

\section{Acknowledgements}

This work has been supported by the Else-Kröner-Fresenius-Stiftung (P65/06// EKMS 06/13; AB), the Deutsche Forschungsgemeinschaft (DFG; BE 4185/1-1; AB), the Nationales Genomforschungsnetz (NGFN; BL), the German Federal Ministry of Education and Research (grant No $01 E 00901$ to the IFBLMU; ME, CL) and the Wellcome Trust and Newcastle University Centre for Brain Ageing and Vitality supported by the Biotechnology and Biological Sciences Research Council, Engineering and Physical Sciences Research Council, Economic and Social Research Council and Medical Research Council (G0700718; DMT). Tissue for this study was provided by the Newcastle Brain Tissue Resource, which is funded in part by grants from the UK Medical Research Council (G0400074; DMT, CM), and the Alzheimer's Society and Alzheimer's Research Trust as part of the Brains for Dementia Research Project. We would like to thank Sebastian Krieg of '3D-bewegungswerkstatt' for the graphic design of figure 5 .

\section{Author details}

${ }^{1}$ Department of Neurology with Friedrich-Baur-Institute, Ludwig-MaximiliansUniversity, 81377 Munich, Germany. ${ }^{2}$ Integrated Center for Research and Treatment of Vertigo, Balance and Ocular Motor Disorders, LudwigMaximilians-University, 81377 Munich, Germany. ${ }^{3}$ Insitute of Applied Physiology, Ulm University, 89081 Ulm, Germany. ${ }^{4}$ Institute for Ageing and 
Health, Newcastle University, Newcastle upon Tyne NE4 5PL, UK. ${ }^{5}$ Medica Toxicology Centre, Wolfson Unit of Clinical Pharmacology, Institute of Neuroscience, Newcastle University, Newcastle upon Tyne NE2 4AA, UK. ${ }^{6}$ Institute of Ageing and Health, Newcastle University Centre for Brain Ageing and Vitality and Mitochondrial Research Group, Newcastle University, Newcastle upon Tyne NE2 4HH, UK. ${ }^{7}$ Experimental Neuropathology, University of California in San Diego (UCSD), La Jolla, CA, 92093, USA. ${ }^{8}$ Institute of Human Genetics, Technical University Munich, 81675 Munich, Germany. ${ }^{9}$ Helmholtz Zentrum Munich, German Research Center for Environmental Health, 85764 Neuherberg, Germany. ${ }^{10}$ Department of Neurology, Therapiezentrum Burgau, 89331 Burgau, Germany.

\section{Authors' contributions}

$A B$, TK and ME designed the study. HP, EM, DMT aided with design details and contributed essential interpretations of findings. CM, BL and FS contributed, characterized and prepared tissue samples for the experiments. SKM, LL, CL and LK performed histochemistry and LMD. ME directly supervised experiments and wrote the paper with assistance of all authors, who have read and approved the final manuscript.

\section{Competing interests}

The authors declare that they have no competing interests.

Received: 20 November 2011 Accepted: 21 December 2011 Published: 21 December 2011

\section{References}

1. Schapira AH, Gegg M: Mitochondrial contribution to Parkinson's disease pathogenesis. Parkinsons Dis 2011, 2011:159160.

2. Harman D: The biologic clock: the mitochondria? J Am Geriatr Soc 1972, 20:145-147.

3. Surmeier DJ, Guzman JN, Sanchez-Padilla J, Goldberg JA: The origins of oxidant stress in Parkinson's disease and therapeutic strategies. Antioxid Redox Signal 2011, 14:1289-1301.

4. Corral-Debrinski M, Horton T, Lott MT, Shoffner JM, Beal MF, Wallace DC Mitochondrial DNA deletions in human brain: regional variability and increase with advanced age. Nat Genet 1992, 2:324-329.

5. Cortopassi GA, Shibata D, Soong NW, Arnheim N: A pattern of accumulation of a somatic deletion of mitochondrial DNA in aging human tissues. Proc Natl Acad Sci USA 1992, 89:7370-7374.

6. Bender A, Krishnan $\mathrm{K}$, Morris $\mathrm{CM}$, Taylor GA, Reeve AK, Perry RH, Jaros $\mathrm{E}_{\text {, }}$ Hersheson JS, Betts J, Klopstock T, Taylor RW, Turnbull DM: High levels of mitochondrial DNA deletions in substantia nigra neurons in aging and Parkinson disease. Nat Genet 2006, 38:515-517.

7. Kraytsberg Y, Kudryavtseva E, McKee AC, Geula C, Kowall NW, Khrapko K: Mitochondrial DNA deletions are abundant and cause functional impairment in aged human substantia nigra neurons. Nat Genet 2006, 38:518-520.

8. Bender A, Schwarzkopf RM, McMillan A, Krishnan KJ, Rieder G, Neumann M, Elstner M, Turnbull DM, Klopstock T: Dopaminergic midbrain neurons are the prime target for mitochondrial DNA deletions. J Neurol 2008, 255:1231-1235.

9. Krishnan KJ, Ratnaike TE, Gruyter HL, Jaros E, Turnbull DM: Mitochondrial DNA deletions cause the biochemical defect observed in Alzheimer's disease. Neurobiol Aging 2011.

10. Bisaglia M, Mammi S, Bubacco L: Kinetic and structural analysis of the early oxidation products of dopamine: analysis of the interactions with alphasynuclein. J Biol Chem 2007, 282:15597-15605.

11. Double KL, Dedov VN, Fedorow H, Kettle E, Halliday GM, Garner B, Brunk UT: The comparative biology of neuromelanin and lipofuscin in the human brain. Cell Mol Life Sci 2008, 65:1669-1682.

12. Fasano M, Bergamasco B, Lopiano L: Modifications of the ironneuromelanin system in Parkinson's disease. J Neurochem 2006, 96:909-916.

13. Zhang W, Phillips $K$, Wielgus AR, Liu J, Albertini A, Zucca FA, Faust R, Qian SY, Miller DS, Chignell CF, Wilson B, Jackson-Lewis V, Przedborski S, Joset D, Loike J, Hong JS, Sulzer D, Zecca L: Neuromelanin activates microglia and induces degeneration of dopaminergic neurons: implications for progression of Parkinson's disease. Neurotox Res 2011, 19:63-72.
14. Zecca L, Casella L, Albertini A, Bellei C, Zucca FA, Engelen M, Zadlo A, Szewczyk G, Zareba M, Sarna T: Neuromelanin can protect against ironmediated oxidative damage in system modeling iron overload of brain aging and Parkinson's disease. J Neurochem 2008, 106:1866-1875.

15. Gerlach M, Double KL, Ben-Shachar D, Zecca L, Youdim MB, Riederer P: Neuromelanin and its interaction with iron as a potential risk factor for dopaminergic neurodegeneration underlying Parkinson's disease. Neurotox Res 2003, 5:35-44.

16. Kastner A, Hirsch EC, Lejeune O, Javoy-Agid F, Rascol O, Agid Y: Is the vulnerability of neurons in the substantia nigra of patients with Parkinson's disease related to their neuromelanin content? J Neurochem 1992, 59:1080-1089.

17. Halliday GM, Ophof A, Broe M, Jensen PH, Kettle E, Fedorow $H$, Cartwright MI, Griffiths FM, Shepherd CE, Double KL: Alpha-synuclein redistributes to neuromelanin lipid in the substantia nigra early in Parkinson's disease. Brain 2005, 128:2654-2664.

18. Fedorow H, Tribl F, Halliday G, Gerlach M, Riederer P, Double KL: Neuromelanin in human dopamine neurons: comparison with peripheral melanins and relevance to Parkinson's disease. Prog Neurobiol 2005, 75:109-124.

19. Oakley AE, Collingwood JF, Dobson J, Love G, Perrott HR, Edwardson JA, Elstner M, Morris CM: Individual dopaminergic neurons show raised iron levels in Parkinson disease. Neurology 2007, 68:1820-1825.

20. Sulzer D: Multiple hit hypotheses for dopamine neuron loss in Parkinson's disease. Trends Neurosci 2007, 30:244-250.

21. Braak H, Del Tredici K, Rub U, de Vos RA, Jansen Steur EN, Braak E: Staging of brain pathology related to sporadic Parkinson's disease. Neurobiol Aging 2003, 24:197-211.

22. Hirsch E, Graybiel AM, Agid YA: Melanized dopaminergic neurons are differentially susceptible to degeneration in Parkinson's disease. Nature 1988, 334:345-348.

23. Chinta SJ, Andersen JK: Redox imbalance in Parkinson's disease. Biochim Biophys Acta 2008, 1780:1362-1367.

24. Guzman JN, Sanchez-Padilla J, Wokosin D, Kondapalli J, lijic E, Schumacker PT, Surmeier DJ: Oxidant stress evoked by pacemaking in dopaminergic neurons is attenuated by DJ-1. Nature 2010, 468:696-700.

25. Elstner M, Morris CM, Heim K, Bender A, Mehta D, Jaros E, Klopstock T, Meitinger T, Turnbull DM, Prokisch H: Expression analysis of dopaminergic neurons in Parkinson's disease and aging links transcriptional dysregulation of energy metabolism to cell death. Acta Neuropathol 2011, 122:75-86.

26. Krishnan K, Reeve AK, Samuels DC, Chinnery PF, Blackwood JK, Taylor RW, Wanrooij S, Spelbrink JN, Lightowlers RN, Turnbull DM: What causes mitochondrial DNA deletions in human cells? Nat Genet 2008, 40:275-279.

27. Mosharov EV, Larsen KE, Kanter E, Phillips KA, Wilson K, Schmitz Y, Krantz DE, Kobayashi K, Edwards RH, Sulzer D: Interplay between cytosolic dopamine, calcium, and alpha-synuclein causes selective death of substantia nigra neurons. Neuron 2009, 62:218-229.

28. Bellinger FP, Bellinger MT, Seale LA, Takemoto AS, Raman AV, Miki $T$ Manning- Bog AB, Berry MJ, White LR, Ross GW: Glutathione Peroxidase 4 is associated with Neuromelanin in Substantia Nigra and Dystrophic Axons in Putamen of Parkinson's brain. Mol Neurodegener 2011, 6:8.

29. Naoi M, Maruyama W, Yi H, Yamaoka Y, Shamoto-Nagai M, Akao Y, Gerlach M, Tanaka M, Riederer P: Neuromelanin selectively induces apoptosis in dopaminergic SH-SY5Y cells by deglutathionylation in mitochondria: involvement of the protein and melanin component. J Neurochem 2008, 105:2489-2500.

30. Li J, Scheller C, Koutsilieri E, Griffiths F, Beart PM, Mercer LD, Halliday G, Kettle E, Rowe D, Riederer P, Gerlach M, Rodriguez M, Double KL: Differential effects of human neuromelanin and synthetic dopamine melanin on neuronal and glial cells. J Neurochem 2005, 95:599-608.

31. Fedorow H, Halliday GM, Rickert CH, Gerlach M, Riederer P, Double KL: Evidence for specific phases in the development of human neuromelanin. Neurobiol Aging 2006, 27:506-512.

32. Saper CB, Petito CK: Correspondence of melanin-pigmented neurons in human brain with A1-A14 catecholamine cell groups. Brain 1982, 105:87-101.

33. Gaspar P, Berger B, Gay M, Hamon M, Cesselin F, Vigny A, Javoy-Agid F, Agid $Y$ : Tyrosine hydroxylase and methionine-enkephalin in the human mesencephalon. Immunocytochemical localization and relationships. J Neurol Sci 1983, 58:247-267. 
34. Fearnley JM, Lees AJ: Ageing and Parkinson's disease: substantia nigra regional selectivity. Brain 1991, 114(Pt 5):2283-2301.

35. Zarow C, Lyness SA, Mortimer JA, Chui HC: Neuronal loss is greater in the locus coeruleus than nucleus basalis and substantia nigra in Alzheimer and Parkinson diseases. Arch Neurol 2003, 60:337-341.

36. McRitchie DA, Cartwright HR, Halliday GM: Specific A10 dopaminergic nuclei in the midbrain degenerate in Parkinson's disease. Exp Neurol 1997, 144:202-213

37. Damier $P$, Hirsch EC, Agid Y, Graybiel AM: The substantia nigra of the human brain. II. Patterns of loss of dopamine-containing neurons in Parkinson's disease. Brain 1999, 122(Pt 8):1437-1448.

38. Gibb WR, Lees AJ: Anatomy, pigmentation, ventral and dorsal subpopulations of the substantia nigra, and differential cell death in Parkinson's disease. J Neurol Neurosurg Psychiatry 1991, 54:388-396.

39. Luoma P, Melberg A, Rinne JO, Kaukonen JA, Nupponen NN, Chalmers RM, Oldfors A, Rautakorpi I, Peltonen L, Majamaa K, Somer H, Suomalainen A: Parkinsonism, premature menopause, and mitochondrial DNA polymerase gamma mutations: clinical and molecular genetic study. Lancet 2004, 364:875-882.

40. Reeve AK, Krishnan KJ, Elson JL, Morris CM, Bender A, Lightowlers RN, Turnbull DM: Nature of mitochondrial DNA deletions in substantia nigra neurons. Am J Hum Genet 2008, 82:228-235.

41. Xuan Q, Xu SL, Lu DH, Yu S, Zhou M, Ueda K, Cui YQ, Zhang BY, Chan P: Increase expression of alpha-synuclein in aged human brain associated with neuromelanin accumulation. J Neural Transm 2011, 118:1575-1583.

42. McKeith IG, Dickson DW, Lowe J, Emre M, O'Brien JT, Feldman H, Cummings J, Duda JE, Lippa C, Perry EK, Aarsland D, Arai H, Ballard CG, Boeve B, Burn DJ, Costa D, Del Ser T, Dubois B, Galasko D, Gauthier S, Goetz CG, Gomez-Tortosa E, Halliday G, Hansen LA, Hardy J, Iwatsubo T, Kalaria RN, Kaufer D, Kenny RA, Korczyn A, et al: Diagnosis and management of dementia with Lewy bodies: third report of the DLB Consortium. Neurology 2005, 65:1863-1872.

43. Krishnan KJ, Bender A, Taylor RW, Turnbull DM: A multiplex real-time PCR method to detect and quantify mitochondrial DNA deletions in individual cells. Anal Biochem 2007, 370:127-129.

doi:

Cite this article as: Elstner et al:: Neuromelanin, neurotransmitter status and brainstem location determine the differential vulnerability of catecholaminergic neurons to mitochondrial DNA deletions. Molecular Brain 2011 4:43.

\section{Submit your next manuscript to BioMed Central and take full advantage of:}

- Convenient online submission

- Thorough peer review

- No space constraints or color figure charges

- Immediate publication on acceptance

- Inclusion in PubMed, CAS, Scopus and Google Scholar

- Research which is freely available for redistribution

Submit your manuscript at www.biomedcentral.com/submit
Biomed Central 